This should be characteristic of the shortest length scales or the fastest physical processes.

To determine if the 'true characteristic' timescales are as long as suggested by the scaling of the 1-second time variability from Cyg X-1, long observations, of roughly 10 days' duration, will be required. As for Cyg X-1, this long timescale is not directly relevant to black-hole models for objects accreting at the Eddington limit. It has been suggested ${ }^{12}$, however, that lowluminosity active galaxies are emitting at $\sim 10^{-3}$ of the Eddington limit; if so, these long timescales are exactly as predicted.

The EXOSAT results have improved $X$-ray timing observations of active galaxies to the point where realistic detailed models are necessary to interpret the data. In some ways this situation is similar to that of brownian motion before its explanation by Einstein. We now have highquality time variability data with no obvious signatures. It is to be hoped that the ASTRO-C observations will greatly increase the database and determine whether X-ray timing measurements can indeed constrain black-hole models of active galaxies.

1. Warwick, R. Proceedings of the Meeting on Accretion onto Compact Objects 1987 (eds Mason, K. O., Watson, M. G \& White, N. E.) 195 (Springer, Berlin, 1987).

2. Lawrence, A., Watson, M. G. Pounds, K. A. \& Elvis, M. Nature 325, 694-696(1987).

3. McHardy, I. \& Czerny, B. Nature 325, 696-698 (1987)

4. Fabian, A. Proc. R. Soc. A366, 499 (1979).

5. Lightman, A. in X-ray Astronomy in the 1980 s (ed. Holt. Lightman, A. in $X$-ray Astronom
S.) 142 (NASA TM 83848,1982 ).

6. Meekins, J. F. et al. Astrophys. J. 278, 288 (1984)

7. Nolan, P. et al. Astrophys. J. 246, 494 (1981).

8. Lawrence, A. Mon. Not. R. astr. Soc. 192, 83 (1980).

9. Mushotzky, R., Holt, S. S. \& Serlemitsos, P. J. Astrophys. J. 255, L115 (1985)

10. Tennant, A. \& Mushotzky, R. F. Astrophys. J. 264, 92 (1983).

11. Barr, P., Clavel, J., Giommi, P. Mushotzky, R. F. \& Madejski, G. in Proc. Conf. Variability Galactic and Extragalactic X-ray Sources (ed. Treves, A.) (Como, Italy, 1986)

12. Barr, P. \& Mushotzky, R. Nature 320, 421 (1986).

13. Lawrence, A., Watson, M., Pounds, K. \& Elvis, M. Mon Not. R. astr. Soc. 217, 685 (1985)

14. Urry, C.M. et al. Proc. Conf. Variability Galactic and Extragalactic Sources (ed. Treves, A.) (Como, Italy, 1986). 15. Mushotsky, R. Adv. Space Res. 13, 157 (1984).

Richard Mushotzky is at the Laboratory for High Energy Astrophysics, NASA Goddard Space Flight Center, Greenbelt, Maryland 20771, USA.

\title{
Analytical geochemistry
}

\section{Surfaces of reacted minerals}

\section{Harold C. Helgeson E.S 7}

UNTIL recently, much of what we know, or think we know, about the mechanisms of interfacial reactions between silicates and aqueous solutions has been derived from subjective interpretation of indirect (and in many cases ambiguous) evidence, largely rate studies of mineral hydrolysis. Now, however, advanced technology is being used to examine and to analyse directly the surfaces of reacted mineral grains to determine the composition of their surface 'skins' and the extent to which they change with reaction progress. These data can be used to infer the stoichiometry of activated complexes at reactive surface sites and to elucidate the mechanisms of surface detachment during the dissolution of complex minerals such as feldspars, pyroxenes and amphiboles. Recent pioneering studies of this kind, one of which is reported by Jean-Claude Petit et al. on page 705 of this issue, use the specific resonant nuclear reaction technique to measure the total hydrogen concentration $\left(\mathrm{H}^{+}, \mathrm{H}_{2} \mathrm{O}\right.$ and $\left.\mathrm{H}_{3} \mathrm{O}^{+}\right)$as a function of depth to about $1,000 \AA$ from the outer surface of reacted diopside $\left(\mathrm{CaMg}\left(\mathrm{SiO}_{3}\right)_{2}\right)$ grains at $\mathrm{pH}$ values of 2 and 6 . The results of Petit et al. indicate that one or more of these species is present in high concentrations at depths of about $500 \AA$ from the outer surface of the reacted diopside grains, and that the concentration is maximal at a depth of about $200 \AA$. Circumstantial evidence indicates that most of the hydrogen is present as
$\mathrm{H}_{2} \mathrm{O}$, but note that small concentrations of $\mathrm{H}_{3} \mathrm{O}^{+}$relative to $\mathrm{H}_{2} \mathrm{O}$ in the hydrogenated surface are sufficient to control the rate of mineral hydrolysis.

The steady-state $p \mathrm{H}$ dependence of mineral dissolution rates observed in the laboratory can be explained in terms of transition-state theory by assuming the presence of activated complexes involving hydrogen, hydronium or hydroxyl ions at the reactive sites. The stoichiometry of these activated complexes depends on the solution $p \mathrm{H}$ and the crystallochemical characteristics of the mineral. For example, recent theoretical consideration by W. M. Murphy (Geochim. cosmochim. Acta, in the press) of pyroxene, wollastonite and olivine dissolution rates reported in the literature indicates that the dissolution stoichometries and $p \mathrm{H}$ dependence of these rates are consistent with steady-state rate control by transition-state decomposition of activated complexes with stoichiometries corresponding to $(\mathrm{H}, \mathrm{C}(2))_{0.5} \mathrm{C}(1)_{0.5} \mathrm{SiO}_{3}^{Z}, \quad\left(\mathrm{H}_{2} \mathrm{Ca}\right) \mathrm{SiO}_{3}$ and $\mathrm{H}(\mathrm{Mg}, \mathrm{Fe})_{2} \mathrm{SiO}_{4}^{+}$, respectively, where $Z$ is the charge on the complex and $\mathrm{C}(1)$ and $\mathrm{C}(2)$ are the cations on the $\mathrm{M}(1)$ and $\mathbf{M}(2)$ octahedral sites, respectively. The resonant nuclear reaction technique and secondary ion mass spectrometry offer hope of direct confirmation of the existence of these species at surface reactive sites such as dislocation outcrops. But much remains to be done in uiscriminate between $\mathrm{H}^{+}, \mathrm{H}_{3} \mathrm{O}^{+}, \mathrm{H}_{2} \mathrm{O}, \mathrm{OH}^{-}$and other species at particular sites. At the moment, only the total concentrations of elements can be measured, and even these are averaged over the surface of the mineral.

In addition to these two techniques, $\mathrm{X}$-ray photoelectron spectroscopy is a powerful method of analysing the surfaces of reactant minerals. This technique has been used to demonstrate that $\mathrm{Ca}$ and $\mathrm{Mg}$ in the $\mathbf{M}(2)$ octahedral sites of dissolving diopside are preferentially released relative to $\mathrm{SiO}_{2}$ from the outermost surface of the reacting mineral (Schott, J., Berner, R. A. \& Sjöberg, E. L. Geochim. cosmochim. Acta 45, 2123-2135; 1981, and Schott, J. \& Berner, R. A. Geochim. cosmochim. Acta 47, 2333-2340; 1983). Comparison of results using all three techniques indicates that the relation between penetration of hydrogen into the surface during dissolution and remobilization of the elements of the reactant mineral at the active surface sites is far more complex for crystalline silicates than for simple silicate glasses.

Studies of reacted mineral surfaces using the three methods I have mentioned above have important implications for many geochemical processes, including weathering, diagenesis and hydrothermal rock alteration. In addition, they should allow better understanding of reactions among ground waters and toxic waste materials, such as solid radioactive waste. Experimental techniques of this kind will help to elucidate the surface chemistry of silicates and hold great promise for unravelling the mechanisms of heterogeneous catalysis on mineral surfaces of both organic and inorganic reactions in geochemical processes. These include decarboxylation reactions and reduction of sulphate in natural waters.

Reactions among mineral surfaces and aqueous solutions is a complex process involving such phenomena as hydration of the surface, adsorption, ion exchange, complex formation, bond disruption and irreversible decomposition of activated complexes followed by surface detachment. Depending on the stoichiometric number per mole of energetically distinct crystallographic sites for cations present in a mineral, which ranges from three in pyroxenes such as diopside to nine in certain amphiboles, many surface species may be involved in the hydrolytic process. In particularly complex minerals such as amphiboles, dissolution mechanisms may involve parallel reactions rather than a simple series of elementary reaction steps. Only when technology is sophisticated enough to detect and unravel these complex mechanisms will we be able to claim with confidence that we can hope to understand the hydrolytic process.

Harold C. Helgeson is Professor of Geochemistry in the Department of Geology and Geophysics, University of California, Berkeley, California 94720 , USA. 